\title{
Left atrial appendage closure in patients with osler disease and atrial fibrillation
}

\author{
Aref Albakri* and Heyder Omran \\ St-Marien hospital Bonn Venusberg, department of internal medicine, Bonn, Germany
}

\begin{abstract}
Background: Treatment of patients with atrial fibrillation (AF) and Osler disease is difficult due to increased risk of bleeding. In these patients, left atrial appendage closure (LAAc) has been shown to be non-inferior to $\mathrm{OAC}$ in preventing thromboembolic events. We present our experience of three clinical cases to assess the safety and efficacy of LAAc and post-procedural single antiplatelet therapy in preventing thrombi formation in Osler patients at 6-months follow-up.

Methods: Three patients, 2 females and 1 male, mean age $64.6 \pm 1.5$, and CHA2DS2-VASc-score $\geq 1$ range 1-3, received LAA closure between 2013 and 2016 . All three had Osler disease and AF with no anomalies or pre-existing LAA thrombi. Under the guidance of TEE, they underwent percutaneous LAA closure with AMPLATZER $^{\mathrm{TM}}$ Amulet ${ }^{\mathrm{TM}}$ device. They received a single antiplatelet therapy with Aspirin or Clopidogrel for six months or until complete device endothelialization. TEE was repeated at six months' follow-up.
\end{abstract}

Findings: Percutaneous LAA closure was successful in all the three patients. At six months' follow-up, while controlling for their other medical situations, there were no peri-procedural complications, bleeding and thrombus formation around the LAA closure device.

Conclusion: Percutaneous LAA closure with post-procedural single antiplatelet therapy presents an alternative to chronic OAC therapy in preventing thromboembolic events in patients intolerant or contraindicated for OAC. Future studies are warranted to assess the indication for LAA closure in patients with Osler disease.

\section{Introduction}

Osler disease is a heterogeneous autosomal dominant hereditary disorder characterized by impaired development of the vasculature, which lack intervening capillaries causing direct connections between arteries and veins [1]. It is a rare disease affecting about one person in 5,000 to 9,000 people [2]. It is rarely symptomatic in children but by the ages of 16 and over 40 years, $70 \%$ and $90 \%$ respectively develop symptoms, which may also appear even as late as 70 years [2]. Its most common clinical manifestations are severe or recurrent epistaxis, small arteriovenous malformations (AVMs), also called telangiectasia, on the nasal, buccal and gastrointestinal (GI) mucosa, and large AVMs in the brain, liver, or lungs [3]. Complications due to hemorrhaging or shunting of blood are usually sudden and life threatening. In a study of 10,293 patients, there were high rates of bleeding complications among hospitalized Osler patients with anemia present in 53\%; epistaxis in $10 \%$; and $38 \%$ received one or more transfusion $[1,2]$. Osler disease has also been associated with elevated Factor VIII with an increased thrombotic risk [4].

Patients with Osler disease who develop atrial fibrillation (AF) present significant clinical challenges in the management of AF. National guidelines: the American College of Cardiology (ACC), the American Heart Association (AHA), the Heart Rhythm Society (HRS) and the National Institute for Health and Care Excellence (NICE) recommend oral anticoagulation (OAC) as the first line medication for the prevention and treatment of thromboembolic events in AF patients [5-7]. However, Osler patients with significant cerebral and pulmonary AVMs have an elevated risk of life-threatening hemorrhaging with relative or absolute contraindication for long-term use of OACs [8]. Intolerance with long-term $\mathrm{OAC}$, and autopsy and echocardiographic data have shown the left atrial appendage (LAA) is a significant source $(<90 \%)$ of cardiac thrombi and thromboembolic complications. This observation led to the development of the strategy of mechanically excluding LAA from systematic circulation [9]. Several recent studies have demonstrated the value of LAA closure as a promising nonpharmacological alternative to OACs for AF patients with an elevated risk of bleeding [10-12].

\section{Current Guidelines for LAA Closure}

Current guidelines for consideration of LAA closure as an intervention for the prevention of stroke varies regionally. The European Society for Cardiology developed class IIb recommendation for percutaneous LAA closure on AF patients with a high risk of stroke and contraindications for chronic OAC [13]. Other indications for LAA closure are AF patients at a high risk of thromboembolic events $\left(\mathrm{CHA}_{2} \mathrm{DS}_{2}-\mathrm{VASc} \geq 2\right.$ ), elevated risk of bleeding (HAS-BLED $\geq 3$ ), or a history of significant intracranial or life-threatening bleeding, and end-stage renal dysfunction (creatinine clearance $<15$ to $30 \mathrm{~mL} / \mathrm{min}$ ). Patients on OAC but having thromboembolic events caused by non$\mathrm{AF}$ related causes such as carotid disease have also been considered for LAA closure [14]. In Canada, LAA closure is indicated to patients with $\mathrm{CHADS}_{2} \geq 1$ and contraindication to long-term OAC. In the

Correspondence to: Aref Albakri, St-Marien hospital Bonn Venusberg, department of internal medicine, Bonn, Germany, E-mail: arefalbakri@yahoo.com

Key words: Osler disease, atrial fibrillation (AF), left atrial appendage (LAA), percutaneous LAA closure, thromboembolic stroke, bleeding, oral anticoagulation $(O A C)$

Received: March 14, 2017; Accepted: April 17, 2017; Published: April 20, 2017 
U.S., it is indicated for patients at high risk of stroke with or without contraindication for OAC [15]. Further, LAA closure should be performed when thrombus has not already developed in the LAA [10].

\section{Safety of LAA Closure}

Clinical techniques for the exclusion of LAA from systemic circulation are percutaneous or surgical. However, surgical removal of LAA is limited because it is invasive and has higher rates of incomplete LAA occlusion associated with an elevated risk of stroke [15]. Further, no study has demonstrated non-inferiority of the surgical approach to OAC. In surgical LAA closure, the continuation of OACs preclude meaningful evaluation of its efficacy [16]. On the other hand, several prospective RCTs have demonstrated percutaneous LAA closure is non-inferior to OACs in the prevention of thromboembolic events in AF patients [17-20]. Further, the efficacy of different devices - PLAATO (no longer available), Amplatzer atrial septal (ASD) and WATCHMANhave been also evaluated [11]. The Watchman (PROTECT AF) clinical trial demonstrated non-inferiority of percutaneous LAA closure to chronic Warfarin in preventing thromboembolic stroke in nonvalvular AF patients with a $\mathrm{CHADS}_{2}$ score of $\geq 1$. Patients who received percutaneous LAA closure had lower end-point of stroke, systemic embolism, and cardiovascular death event rates (3.0) compared to Warfarin (4.3) per 100 patient-years, and had statistically lower significant disability or death [11]. While safety events in Warfarin patients had a constant occurrence over time, LAAc patients had higher initial event rate because of incomplete device endothelialization but with a constant rate during the follow-up period [19]. However, the safety events for LAAc patients were largely procedure-related, which reduced in intensity as operator efficiency increased [11].

Perrotta et al. [10]. studied complications (safety events) associated with percutaneous LAA closure and categorized them into three groups: access-related, device-related and antithrombotic-treatment related. Access-related events are vascular complications predominately groin hematoma, femoral arterial pseudo aneurysm or arteriovenous fistula, which often necessitate transfusion or surgical procedures. Devicerelated events are trans septal LA access associated with the risk of air embolism and pericardial effusion while implantation events are device dislodgement and incomplete LAA closure with residual peri-device blood flow. Antithrombotic treatment related events are associated with post-operation treatment to prevent thrombus formation [10].

\section{Methods and procedure}

\section{Device description}

We used St. Jude Medical (SJM) AMPLATZER ${ }^{\mathrm{TM}}$ Amulet $^{\mathrm{TM}}$ device to perform LAAc. The device is the second generation of the AMPLATZER $^{\mathrm{TM}}$ cardiac plug (ACP) device. It is a self-expanding nitinol stent providing double device closure. Its major components are distal lobe (diameter range $16-34 \mathrm{~mm}$ ) with 6-10 pairs of stabilizing wires and a proximal disc (Figure 1). The lobe anchors the device and occludes the LAA at the neck $12-15 \mathrm{~mm}$ from the ostium while the disc occludes the LAA ostium at the atrial side. The device also has a proximal screw, which when recessed, minimizes formation of thrombus on the proximal disc and facilitate device re-attachment to the pusher screw. The device is suitable for LAA neck diameters 11-31 $\mathrm{mm}$ and depth $\geq 12 \mathrm{~mm}$ [21].

\section{Patient selection}

Initially, we determine the baseline clinical characteristics of patients for indications for LAAc. Non-valvular AF and a history of

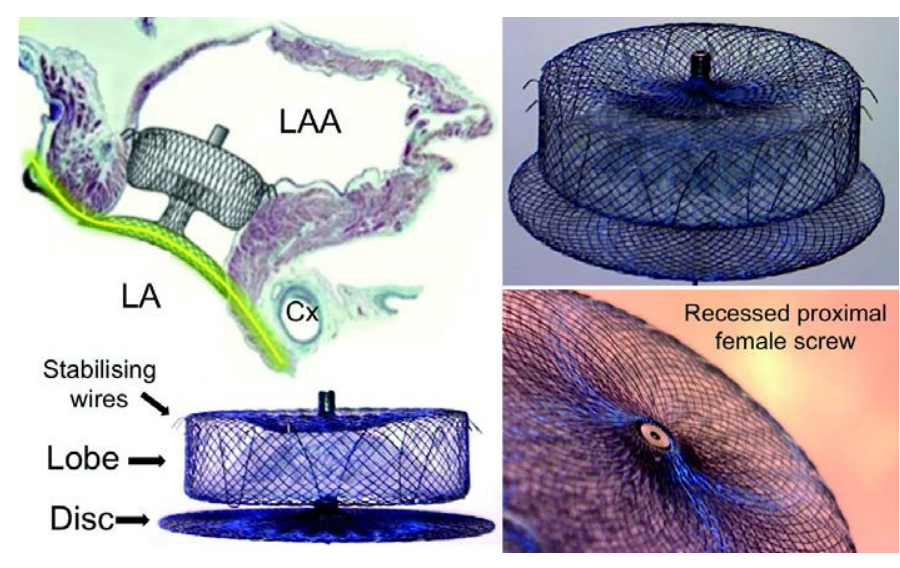

Figure 1. AMPLATZER ${ }^{\mathrm{TM}}$ Amulet $^{\mathrm{TM}}$ Device [19].

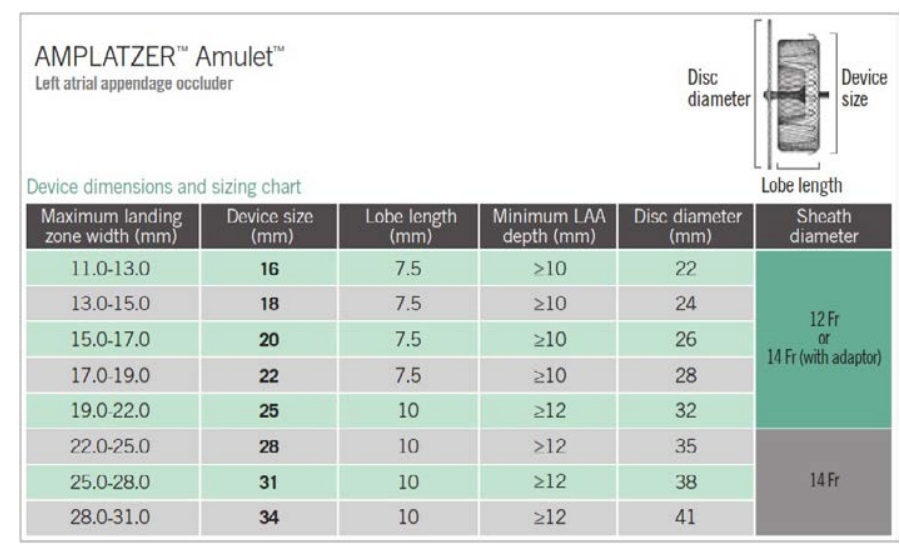

Figure 2. Device Dimension and Sizing Chart for AMPLATZER Amulet Device [19].

bleeding or elevated risk of bleeding requiring OAC therapy is the most common indications for LAAc. We performed baseline transesophageal echocardiographic (TEE) to exclude pre-existing thrombi in LAA, which is the most important contraindication for LAAc [15]. If thrombus was present in the LAA, OAC therapy was recommended for four weeks or until the thrombi dissolves. We also used the baseline TEE to detect pericardial effusion and to assess the function of mitral valve and patency of the left upper pulmonary vein [21]. We also checked for other contraindications for LAAc with AMPLATZER Amulet device such as presence of infective endocarditis, extreme LAA sizes, extensive congenital cardiac anomaly only treated by surgery, sepsis or untreated ulcer a month to implantation, bleeding disorder, untreated ulcer, contraindications for Aspirin or Clopidogrel, pregnancy and device interference with other inter-cardiac structures [15].

\section{Procedure}

The procedure for LAAc was performed as described by Earley and Tzikas et al. [21,22]. under TEE guidance and anesthesia. We obtained trans septal access in the right femoral vein through a 3-4 mm skin incision using a SJM BRK-1 ${ }^{\mathrm{TM}}$ needle, which has been shown to provide support and proper angulation for puncture. Patients were administered unfractionated heparin in two doses-before and after a successful trans septal puncture-to maintain activated clotting time $>250$ seconds and to provide sufficient antithrombotic protection in the case of a prolonged procedure. We flushed the catheter regularly with heparinized saline to avoid thrombi formation in the wires or catheter within the LA thrombogenic environment. We then performed CT angiography to define the exact LAA size (the maximum and minimum 
diameter of LAA ostium and landing zone) and shape (circular or oval). We selected a delivery sheath based on the chosen device size, 1-2 mm more than the corresponding dimension on the sizing chart (Figure 2). We exchanged transseptal sheath and delivery sheath in the left upper pulmonary vein. Under fluoroscopy and TEE guidance, we moved delivery sheath across the atrial septum and deployed to the landing zone. The device opened up like a ball with a disc covering the OS of the LAA and implanted in the LAA. After implantation, we evaluated device position, peri-device leak and stability using TEE. Multiple device views, color Doppler signals and contract media injection to visualize and exclude peri-device leak and confirm the position of the device. Additional tug test, pulling the device disc from the lobe for 20 30 seconds, tested device stability.

During and post-procedure, we examined patients for device and procedure-related risks. These risks included allergic reaction to device; damage to vein; pain, hematoma or seroma at insertion site; hemolysis, infection at insertion site or in the heart, thrombus formation, cardiac arrhythmia, hemorrhaging, air emboli, device dislodgement or migration, perforation of cardiac structures and incomplete LAA closure. After successful device implantation, patients were put under a single antiplatelet therapy with Aspirin or Clopidogrel for 6 months to prevent device-related thrombus formation until device endothelialization. In about 45 days, a thin layer of tissues grows around the device and the device prevents thrombus in the LAA from entering systemic circulation.

\section{Follow-up}

Following the successfully implantation of the AMPLATZER Amulet device, patients were prescribed with endocarditis prophylaxis and a single antiplatelet therapy (Aspirin or Clopidogrel) for six months. The goal of the prescription was to prevent infection and thrombus formation around the AMPLATZER Amulet device while allowing the body to adopt to the new device. The decision to continue endocarditis prophylaxis beyond six months was the discretion of the physician. Post-procedural follow-up was performed at six months under TEE guidance to assess LAA closure, formation of thrombus, the position of the device and any defect allowing peri-device flow.

\section{Clinical cases}

Three patients (Patient 1: female, Patient 2: male and Patient 3: female) aged 66, 63 and 65 years received percutaneous LAA closure in 2013, 2015 and 2016 respectively. All the three patients had been documented with Osler disease, atrial fibrillation (AF) and had elevated risks for thromboembolic stroke $\left(\mathrm{CHA}_{2} \mathrm{DS}_{2}\right.$-VASc-score 3, 1 , 3 ) respectively. Further clinical tests revealed none of the three patients had any anomaly or any detectable pre-existing LAA thrombus.
The baseline clinical characteristics for Patient 1 was nasopharynx bleeding after using Xarelto $15 \mathrm{mg}$ per day. She underwent topical intranasal treatment with tranexamic acid prior to the procedure. She had hypertension, left ventricular (LV) hypertrophy II, heart failure, and degenerative changes in ascending and descending aorta $4 \mathrm{~mm}$. Patient 2 had concentric LV hypertrophy II, high grade pulmonary hypertension, and left renal nephrectomy after renal carcinoma. Patient 3 had many lesions in the nose, which contraindicated long-term use of OAC, aortic valve insufficiency by Valsalva sinus aneurysm grade III, mitral valve insufficiency grade II, huge intrahepatic AV shunt and Gilbert disease (Table 1 summarizes the baseline characteristics of the three patients).

\section{Procedural results}

Before LAA device implantation, all the three patients underwent TEE to assess the anatomy of LAA and to exclude pre-existing formation of LAA thrombus. The LAA closure was performed in all the three patients as outlined in the method and procedure section. After LAA closure, all the three patients received maintenance doses with antiplatelet aggregation, Clopidogrel for Patient 1 and 3, and Aspirin for Patient 2, for six months to prevent thrombus formation around the device before complete endothelialization (the body to adapt to the new device). Percutaneous LAA closure was successful in all the three patients. There were no reported peri-procedural complications. Aside from Patient 1, who had epistaxis post LAAc where bleeding was well controlled, all the three patients coped well with the AMPLATZER Amulet LAA device.

\section{Follow-up results}

At six months' follow-up, in all the three patients, while controlling for their other medical situations, TEE results showed the LAA closure was successful without any complications. There were no thromboembolic events, no thrombus formation around the LAA closure device and no residual peri device leaks.

\section{Discussion}

The treatment of Osler disease and AF patients is difficult due to high risk of bleeding under OAC. Two case studies published in 2011 and 2012 demonstrated the feasibility of AMPLATZER cardiac plug device plug and the Watchman device. In contrast to the Watchman LAA occlusion device, post intervention OAC is not required after the procedure using the AMPLATZER cardiac plug device. In our study, the primary endpoint was the safety and efficacy of percutaneous LAAc with the AMPLATZER Amulet device in the prevention of thromboembolic events in patients with AF and Osler disease at a mid-term (sixmonth) follow-up. The secondary endpoint was the effectiveness of post-procedural single antiplatelet therapy as a non-pharmacological

Table 1. Baseline characteristics.

\begin{tabular}{|c|c|c|c|}
\hline Characteristics & Patient 1 & Patient 2 & Patient 3 \\
\hline Gender & Female & Male & Female \\
\hline Age (in years) & 66 & 63 & 65 \\
\hline $\mathrm{CHA}_{2} \mathrm{DS}_{2}$-VASc-score & 3 & 1 & 3 \\
\hline Anomalies & None & None & None \\
\hline Thrombus & None & None & None \\
\hline Medical stand & $\begin{array}{l}\text { AF, Osler disease, hypertension, LV hypertrophy } \\
\text { II, heart failure, degenerative changes in ascending } \\
\text { and descending aorta }(4 \mathrm{~mm} \text { calc), nasopharynx } \\
\text { bleeding after Xarelto } 15 \mathrm{mg} / \text { day }\end{array}$ & $\begin{array}{l}\text { AF, Osler disease. Hypertension, } \\
\text { concentric LV hypertrophy II, high- } \\
\text { grade pulmonary hypertension, } \\
\text { left renal nephrectomy after renal } \\
\text { carcinoma. }\end{array}$ & $\begin{array}{l}\text { AF, Osler disease, aortic valve } \\
\text { insufficiency by valsalva sinus } \\
\text { aneurysm grade III, mitral valve } \\
\text { insufficiency grade II, huge } \\
\text { intrahepatic AV shunt, Gilbert disease. }\end{array}$ \\
\hline
\end{tabular}


alternative to OAC. Under a single antiplatelet therapy, we investigated three patients with a risk of thromboembolic stroke $\left(\mathrm{CHA}_{2} \mathrm{DS}_{2}-\mathrm{VASc}\right.$ score $\geq 1$ ) undergoing LAAc. One patient had multiple lesions in the nose and another did not use OAC because of recurrent epistaxis after Xarelto use (15 mg/day). At six months' post LAAc, we found no perioperative complications, bleeding complications, per-device leaks, thrombus formation around the LAAc device and all the three patients coped with the AMPLATZER Amulet device.

In our three clinical cases, we found no thrombi on the device after 6 months' follow-up, which supports the observations by Tzikas et al. [21] that the AMPLATZER Amulet device could eliminate the need for OAC therapy for the prevention of thrombus formation. All our three patients with Osler disease received either Aspirin or Clopidogrel. Initial instructions for use (IFU) recommends antiplatelet therapy for 6 months to prevent the thrombus formation. Long-term treatment is not necessary after LAAc. This is of particular importance in Osler patients since bleeding risk may be elevated under antiplatelet therapy. However, further investigation is warranted to justify whether LAAc is an effective alternative treatment for the OAC in patients intolerant to antiplatelet therapy. One of our patients with repeated bleeding underwent topical treatment with tranexamic acid prior to the procedure. We observed no peri-procedural complication. Hence, optimal patient preparation is recommended in particular patients who tend to bleed easily. Out of the two patients with recurrent epistaxis, there was no hospitalization after successful LAAc during follow-up.

Our findings are consistent with those of Fatkin, Kelly and Feneley, and Blackshear and Odell $[23,24]$, who were the first to suggest the value of LAA closure as a prophylaxis against stroke in AF patients. Sick et al. and Sievert et al. $[25,26]$ also demonstrated mechanical exclusion of the LAA from systemic circulation reduces thrombi formation and thromboembolic complications in AF patients. Recently, two followup sub studies on PROTECT AF $[11,19]$ also support our findings. The two studies demonstrated non-inferiority of LAAc to OAC in preventing thromboembolic events, superiority to Warfarin for cardiovascular and all-cause mortality and a significant reduction in procedure and device-related complications in LAAc. The two studiers associated fewer device and procedure-related risks in LAAc with improvement in operative experience [27]. observation study on the Lariant device also finds LAA closure has low access complications and peri-procedural adverse events while Devlin et al. [28]. found antiplatelet aggregation therapy more valuable than OAC for Osler patients after undergoing percutaneous LAA closure. However, Lam, et al. [29] associate incomplete LAA closure with an increased risk of LAA thrombus formation through peri-device flow. With supporting evidence for LAAc in AF patients and Osler growing, research interest is expanding to evaluate alternatives to OAC for the prevention of post-procedural thrombi formation. With no clear guidelines existing on alternative pharmacological therapy to $\mathrm{OAC}$ and on the duration of the therapy, the prevention of thrombus remains important in the first few months after LAAc procedure and before complete device endothelialisation. Additionally, no studies have examined LAAc without anticoagulation or antiplatelet therapy to indicate the necessity for antiplatelet therapy. In our study, we find a single antiplatelet therapy with Aspirin or Clopidogrel for six months was successful in the prevention of post-procedural thrombi formation.

Our findings support those of three earlier clinical case reports $[30,31,12]$, but with variations in the duration of antiplatelet therapy. In the case report by Brenna et al. [30], a 71-year old patient with Osler and AF underwent LAAc and post-procedural Clopidogrel therapy for one month. Control TEE showed no peri-device leaks and thrombus formation. Nine months later, there were reported no complications. In case report [31], a 79-year old Osler and AF patient received Aspirin for six weeks after undergoing LAAc. Controlled TEE at 45 days' post-procedure revealed successful LAA occlusion and no thrombus formation, and Aspirin stopped. Finally, in the case report by Vorselaars et al. [12], which involved five patients, three continued OAC therapy, one received Aspirin therapy and the other a dual therapy of Aspirin and Clopidogrel. At three months' follow-up, there was no thrombotic complications. The patient on Aspirin had residual flow and stopped Aspirin while the other four switched to a single Aspirin therapy. At 12-month follow-up, there were no reports of thrombus formation.

\section{Conclusion}

Patients with AF and Osler disease have a high risk of bleeding and usually contraindicated for OAC. In these patients, LAAc provides an alternative treatment to OACs for preventing thromboembolic events. Post procedural single antiplatelet therapy with Aspirin or Clopidogrel is safe and efficacious in preventing thrombus formation until device endothelialization. Further large-scale randomized controlled trials are warranted to assess the indication for LAAc in patients with Osler disease and the safety of post-procedural single antiplatelet therapy for thrombus prevention.

\section{References}

1. McDonald J, Pyeritz RE (2017) Hereditary Hemorrhagic Telangiectasia. In Pagon, RA, Adam MP, Ardinger HH, et al. (Eds). Seattle: University of Washington, pp. 19932017.

2. Rimmer J, Lund VJ (2015) Hereditary hemorrhagic telangiectasia. Rhinology 53: 195 203. [Crossref]

3. McDonald J, Bayrak-Toydemir P, \& Pyeritz RE (2011) Hereditary hemorrhagic telangiectasia: An overview of diagnosis, management, and pathogenesis. Genet Med 13: 607-616. [Crossref]

4. Shovlin CL, Sulainam NL, Govani FS, Jackson JE, \& Begbie ME (2007) Elevated factor VIII in hereditary hemorrhagic telangiectasia (HHT): Association with venous thromboembolism. Thromb Haemost 98: 1031-9. [Crossref]

5. January CT, Wann LS, Alpert JS, Calkins H, Cigarroa JE, et al. (2014) 2014 AHA ACC/HRS guideline for the management of patients with atrial fibrillation: executive summary: a report of the American College of Cardiology/American Heart Association Task Force on practice guidelines and the Heart Rhythm Society. Circulation 130 2071-104. [Crossref]

6. National Institute for Health and Care Excellence. (2014) Atrial fibrillation management: Clinical guidelines, 18 June 2014.

7. Nishimura RA, Otto CM, Bonow RO, Carabello BA, Erwin JP, et al. (2014) 2014 AHA ACC Guideline for the Management of Patients with Valvular Heart Disease: executive summary: a report of the American College of Cardiology/American Heart Association Task Force on Practice Guidelines. Circulation 129: 2440-92. [Crossref]

8. Faughnan ME, Palda VA, Garcia-Tsao G, Geisthoff UW, McDonald J, et al. (2011) International guidelines for the diagnosis and management of hereditary hemorrhagic telangiectasia. J Med Genet 48: 73-87. [Crossref]

9. Swaans MJ, Post MC, Rensing BJWM, and Boersma LVA (2012). Percutaneous left atrial appendage closure for stroke prevention in atrial fibrillation. Neth Heart $J$ 20: 161-166. [Crossref]

10. Perrotta L, Bordignon S, Dugo D, Fürnkranz A, Konstantinou A, et al. (2014) Complications from left atrial appendage exclusion devices. J Atr Fibrillation 7: 66-72. [Crossref]

11. Reddy VY, Holmes D, Doshi SK, Neuzil P, Kar S (2011) Safety of percutaneous left atrial appendage closure: results from the Watchman Left Atrial Appendage System for Embolic Protection in Patients with AF (PROTECT AF) clinical trial and the Continued Access Registry. Circulation 123: 417-424. [Crossref]

12. Vorselaars VM, Velthuis S, Swaans MJ, Mager JJ, Snijder RJ, et al. (2015) Percutaneous 
left atrial appendage closure: An alternative strategy for anticoagulation in atrial fibrillation and hereditary hemorrhagic telangiectasia. Cardiovasc Diagn Ther 5: 4953. [Crossref]

13. Camm AJ, Lip GY, De Caterina R, Savelieva I, Atar D, et al. (2012) 2012 focused update of the ESC Guidelines for the management of atrial fibrillation: an update of the 2010 ESC Guidelines for the management of atrial fibrillation. Developed with the special contribution of the European Heart Rhythm Association. Eur Heart $J$ 33: 2719-47. [Crossref]

14. Pison L, Potpara TS, Chen J, Larsen TB, Bongiorni MG, et al. (2015) Left atrial appendage closure-indications, techniques, and outcomes: Results of the European Heart Rhythm Association Survey. Europace 17: 642-6. [Crossref]

15. Saw J, Lempereur M (2014) Percutaneous left atrial appendage closure: procedural techniques and outcomes. JACC Cardiovasc Interv 7: 1205-1220. [Crossref]

16. Nietlispach F, Glokler S, Khattab A, Pilgrim T, Schmid M, et al. (2012) Percutaneous left atrial appendage closure. European Geriatric Medicine 3: 308-311.

17. Holmes DR, Reddy VY, Turi ZG, Doshi SK, Sievert H, et al. (2009) Percutaneous closure of the left atrial appendage versus warfarin therapy for prevention of stroke in patients with atrial fibrillation: A randomised non-inferiority trial. Lancet 374: 534-542. [Crossref]

18. Park JW, Bethencourt A, Sievert H, Santoro G, Meier B, et al. (2011) Left atrial appendage closure with Amplatzer cardiac plug in atrial fibrillation: initial European experience. Catheter Cardiovasc Interv 77: 700-706. [Crossref]

19. Reddy VY, Sievert H, Halperin J, Doshi SK, Buchbinder M, et al. (2014) Percutaneous left atrial appendage closure vs warfarin for atrial fibrillation: a randomized clinical trial. JAMA 312: 1988-1998. [Crossref]

20. Viles-Gonzalez JF, Kar S, Douglas P, Dukkipati S, Feldman T, et al. (2012) The clinical impact of incomplete left atrial appendage closure with the Watchman Device in patients with atrial fibrillation: a PROTECT AF (Percutaneous Closure of the Left Atrial Appendage Versus Warfarin Therapy for Prevention of Stroke in Patients with Atrial Fibrillation) substudy. J Am Coll Cardiol 59: 923-929. [Crossref]
21. Tzikas A, Gafoor S, Meerkin D, Freixa X, Cruz-Gonzalez I, et al. (2016) Left atrial appendage occlusion with the AMPLATZER Amulet device: an expert consensus stepby-step approach. EuroIntervention 11: 1512-1521. [Crossref]

22. Earley MJ (2009) How to perform a transseptal puncture. Heart 95: 85-92. [Crossref]

23. Fatkin D, Kelly R, and Feneley MP (1994) Left atrial appendage blood velocity and thromboembolic risk in patients with atrial fibrillation. $J$ Am Coll Cardiol 24: 14291430. [Crossref]

24. Blackshear JL and Odell JA (1996) Appendage obliteration to reduce stroke in cardiac surgical patients with atrial fibrillation. Ann Thorac Surg 61: 755-759. [Crossref]

25. Sick PB, Schuler G, Hauptmann KE, Grube E, Yakubov S, et al. (2007) Initia worldwide experience with the WATCHMAN left atrial appendage system for stroke prevention in atrial fibrillation. J Am Coll Cardiol 49: 1490-1495. [Crossref]

26. Sievert H, Lesh MD, Trepels T, Omran H, Bartorelli A, et al. (2002) Percutaneous lef atrial appendage transcatheter occlusion to prevent stroke in high-risk patients with atrial fibrillation: early clinical experience. Circulation 105: 1887-1889. [Crossref]

27. Bartus K, Han FT, Bednarek J, Myc J, Kapelak B, et al. (2013) Percutaneous left atrial appendage suture ligation using the LARIAT device in patients with atrial fibrillation: initial clinical experience. J Am Coll Cardiol 62: 108-118. [Crossref]

28. Devlin HL, Hosman AE, and Shovlin CL (2013) Antiplatelet and anticoagulant agents in hereditary hemorrhagic telangiectasia. $N$ Engl J Med 368: 876-8. [Crossref]

29. Lam SCC, Bertog S, and Sievert H (2015) Incomplete left atrial appendage occlusion and thrombus formation after Watchman implantation treated with anticoagulation followed by further transcatheter closure with a second-generation Amplatzer Cardiac Plug (Amulet device). Catheter Cardiovasc Interv 85: 321-327. [Crossref]

30. Brenna E J, Bethencourt A, Contreras A E, Ballarino MA, Peirone A (2011) Percutaneous Left Atrial Appendage Closure in a Patient with Hereditary Hemorrhagic Telangiectasia and Atrial Fibrillation. Texas Heart Institute Journal 38: 604-605.

31. Velthuis S, Swaans MJ, Mager JJ, Rensing BJ, Boersma LV, et al. (2012) Left atrial appendage closure for stroke prevention in patients with atrial fibrillation and hereditary hemorrhagic telangiectasia. Case Rep Cardiol: 646505. [Crossref]

Copyright: (C2017 Albakri A. This is an open-access article distributed under the terms of the Creative Commons Attribution License, which permits unrestricted use, distribution, and reproduction in any medium, provided the original author and source are credited. 